\title{
Adaption of the Michelson interferometer for a better understanding of the temporal coherence in lasers
}

M. Illarramendi, J. Zubia, J. Arrue, I. Ayesta

M. A. Illarramendi, J. Zubia, J. Arrue, I. Ayesta, "Adaption of the Michelson interferometer for a better understanding of the temporal coherence in lasers," Proc. SPIE 10452, 14th Conference on Education and Training in Optics and Photonics: ETOP 2017, 1045249 (16 August 2017); doi: 10.1117/12.2266546

EDent: 14th Conference on Education and Training in Optics and Photonics, ETOP 2017, 2017, Hangzhou, China 


\title{
Adaption of the Michelson Interferometer for a better understanding of the temporal coherence in lasers
}

\author{
M.A. Illarramendi ${ }^{a}$, J. Zubia ${ }^{b}$, J. Arrue $^{\mathrm{b}}$, and I. Ayesta ${ }^{\mathrm{c}}$ \\ ${ }^{a}$ Applied Physics Department I, ${ }^{b}$ Communications Engineering Department, ${ }^{\mathrm{c}}$ Applied Mathematics \\ Department \\ Faculty of Engineering. University of the Basque Country UPV/EHU. \\ Plaza Torres Quevedo 1, 48013 Bilbao (Spain).
}

\begin{abstract}
In this work, we show a design of a laboratory exercise in which a digital camera has been coupled to a Michelson interferometer based on free-propagation arms. By using the camera, our students measure the evolution of the interference patterns as a function of the difference between the optical paths of the arms. In this way, they obtain the corresponding reduction of the contrast of the fringes. The analysis of the results allows one to calculate the coherence length, and also to relate the temporal coherence of the employed laser with its spectral line profile. The exercise has been carried out with two lasers, which present different coherence lengths.
\end{abstract}

Keywords: Michelson interferometer, temporal coherence, fringe visibility, image processing

\section{INTRODUCTION}

Light coherence plays an important part in modern optics. In fact, it is one of the properties that distinguish lasers from other light sources. Light coherence is very important in applications such as holography, some types of spectroscopy, information recording and processing, optical communications, and so on. Since the phenomenon of light coherence is both space and time dependent, we need to draw a distinction between temporal and spatial coherence. Spatial coherence refers to the phenomenon whereby the phase difference between two points in a wavefront of an electromagnetic field remains constant with time. This can be measured by using wavefront-splitting interferometers like the Young's slit interferometer. On the other hand, temporal coherence occurs when the phase difference between two time instants at a given point remains constant with time. Temporal coherence is intimately related to interferometry and it is usually measured by means of a Michelson interferometer, which is an interferential device based on the division of light amplitude. It consists in dividing the incident beam into two beams and rejoining them after travelling different paths. The two beams produce interference that varies with the difference between the distances travelled along the two paths. The existence of interference fringes is a manifestation of the temporal coherence between the interfering beams, which can be explained from the time delay of one beam with respect to the other one due to their optical paths being different.

Although the basic concepts of light coherence are introduced in most undergraduate optics textbooks [1-3], there are relatively few experiments to be carried out as laboratory exercises in which students can gain insight into the practical concepts related to the distinction between temporal and spatial coherence and concepts such as coherence time, and coherence length. In this work, we detail a laboratory exercise that serves for a better understanding of the concept of temporal coherence of light sources. This has been developed within the course of Space Interferometry pertaining to the Master in Space Science and Technology at the University of the Basque Country (UPV / EHU) [4]. A Michelson interferometer, to which a digital camera has been coupled, is used to quantitatively analyze the phenomenon of interference from various sources of excitation. In particular, two different lasers have been employed: a He-Ne red laser and a diode-pumped solid state green laser. We determine the variation of the fringe visibility as a function of the path difference by changing the length of one arm. The spectral line profiles of the two lasers are compared and analyzed. In

14th Conference on Education and Training in Optics and Photonics: ETOP 2017, edited by Xu Liu,

Xi-Cheng Zhang, Proc. of SPIE Vol. 10452, 1045249 - ( 2017 ICO, IEEE, OSA, SPIE

CCC code: $0277-786 X / 17 / \$ 18 \cdot$ doi: $10.1117 / 12.2266546$ 
the following sections we describe the theoretical foundations, then the experimental design and finally the results of the experiment.

\section{THEORY}

\subsection{The Michelson interferometer}

In the Michelson interferometer a beam from a laser is divided by a beam splitter (see Fig. 1). One of the beams is reflected on a fixed mirror (M1) and the other on a movable mirror (M2). Both beams return to a detector where the beams are superposed. Interference fringes corresponding to the two light beams can be observed on the detector plane. According to the superposition principle, the oscillating electric fields of the two light beams are added at each point of the detector plane, i.e. $E=E_{1}+E_{2}$. As a consequence, the intensity (or irradiance) $I$ of the light, or light power per unit area, is expressed as:

$$
I=\left\langle|E|^{2}\right\rangle=\left\langle\left|E_{1}+E_{2}\right|^{2}\right\rangle I_{1}+I_{2}+2|\gamma| \sqrt{I_{1} I_{2}} \cos \varphi
$$

where $\left.\varphi=\arg \left(<E_{1} E_{2}^{*}\right\rangle\right), I_{1}$ and $I_{2}$ are the values of the irradiances of the superposed beams and $\gamma$ is the degree of coherence between $E_{1}$ and $E_{2}$, defined as:

$$
\gamma=\frac{\left\langle E_{1} E_{2}^{*}\right\rangle}{\sqrt{\left\langle\left|E_{1}\right|^{2}\right\rangle\left\langle\left|E_{2}\right|^{2}\right\rangle}}
$$

$\gamma$ measures the degree of temporal coherence between the two beams, since $E_{1}$ and $E_{2}$ in Eq. (2) represent the values of the electric fields on the same point $x$ at two different instants of time $t_{1}$ and $t_{2}$, i.e. $E_{1}=E\left(x, t_{1}\right)$ and $E_{2}=E\left(x, t_{2}\right)$.

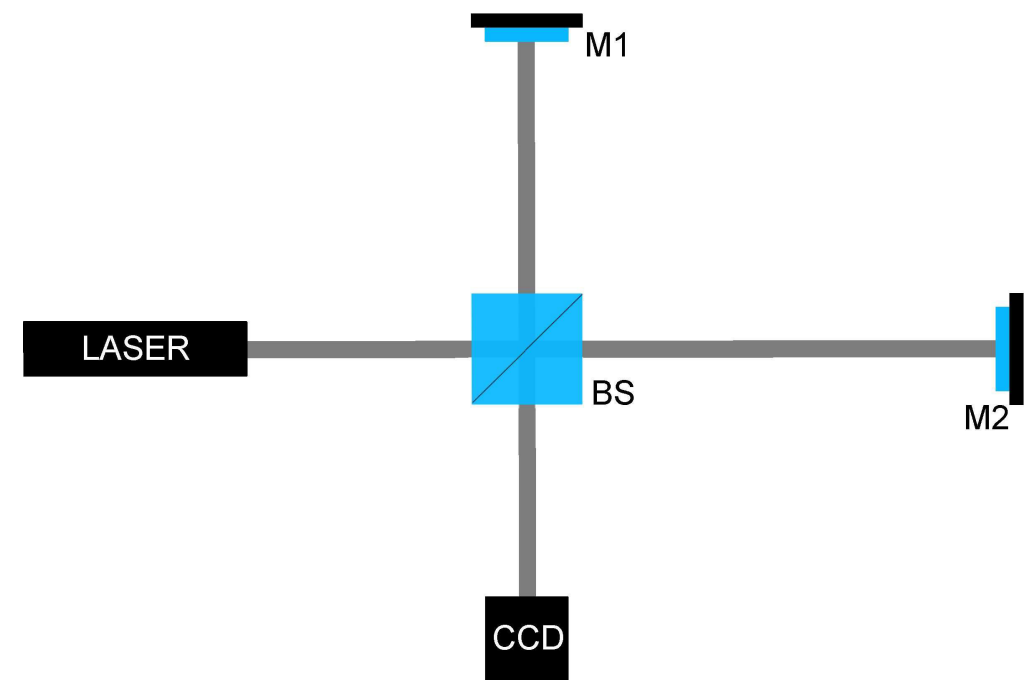

Figure 1. Schematic of the Michelson interferometer. M1: fixed mirror; M2: movable mirror; BS: Beam splitter; CCD: digital camera.

The quality of the interference fringes can be described quantitatively in terms of the fringe visibility $V$, which is defined as:

$$
V=\frac{I_{\max }-I_{\min }}{I_{\max }+I_{\min }}
$$


where $I_{\max }$ and $I_{\min }$ are the maximum and minimum values of the irradiance in the interference pattern. The visibility is related to the module of $\gamma$ which, in the case of a typical Michelson interferometer having equal irradiances in the superposed beams, can be expressed as:

$$
V=\frac{2|\gamma| \sqrt{I_{1} I_{2}}}{I_{1}+I_{2}}=|\gamma|
$$

That is to say, the fringe visibility is equal to the modulus of $\gamma$. In the case of complete coherence $(|\gamma|=1)$, the interference fringes have the maximum contrast $V=1$, whereas, for complete incoherence $(|\gamma|=0)$, the visibility is 0 , i.e. there are no interference fringes.

\subsection{Wiener-Khinchin theorem}

There is a relation between the degree of temporal coherence $(\gamma)$ and the spectral irradiance distribution of the light source, $I(\omega)$. If we consider a light beam as a sum (or integral) of monochromatic waves, we have:

$$
V(\tau)=|\gamma(\tau)|=\frac{\left\langle E_{1} E_{2}^{*}\right\rangle}{I}=\frac{\sum_{k}\left|E_{0, k}\right|^{2} \exp \left[i\left(\omega_{k} \tau\right)\right]}{I}=\frac{\int I(\omega) \exp (i \omega \tau) d \omega}{I}
$$

where $\tau=t_{2}-t_{1}$ and $I\left(\omega_{k}\right) \sim\left|E_{0, k}\right|^{2}$ expresses the irradiance of each monochromatic component of the light beam. This result, known as Wiener-Khinchin theorem, indicates that the degree of temporal coherence is given by the inverse Fourier transform of the spectral distribution of the light beam.

Table I. Spectral distributions and their visibility expressions and curves

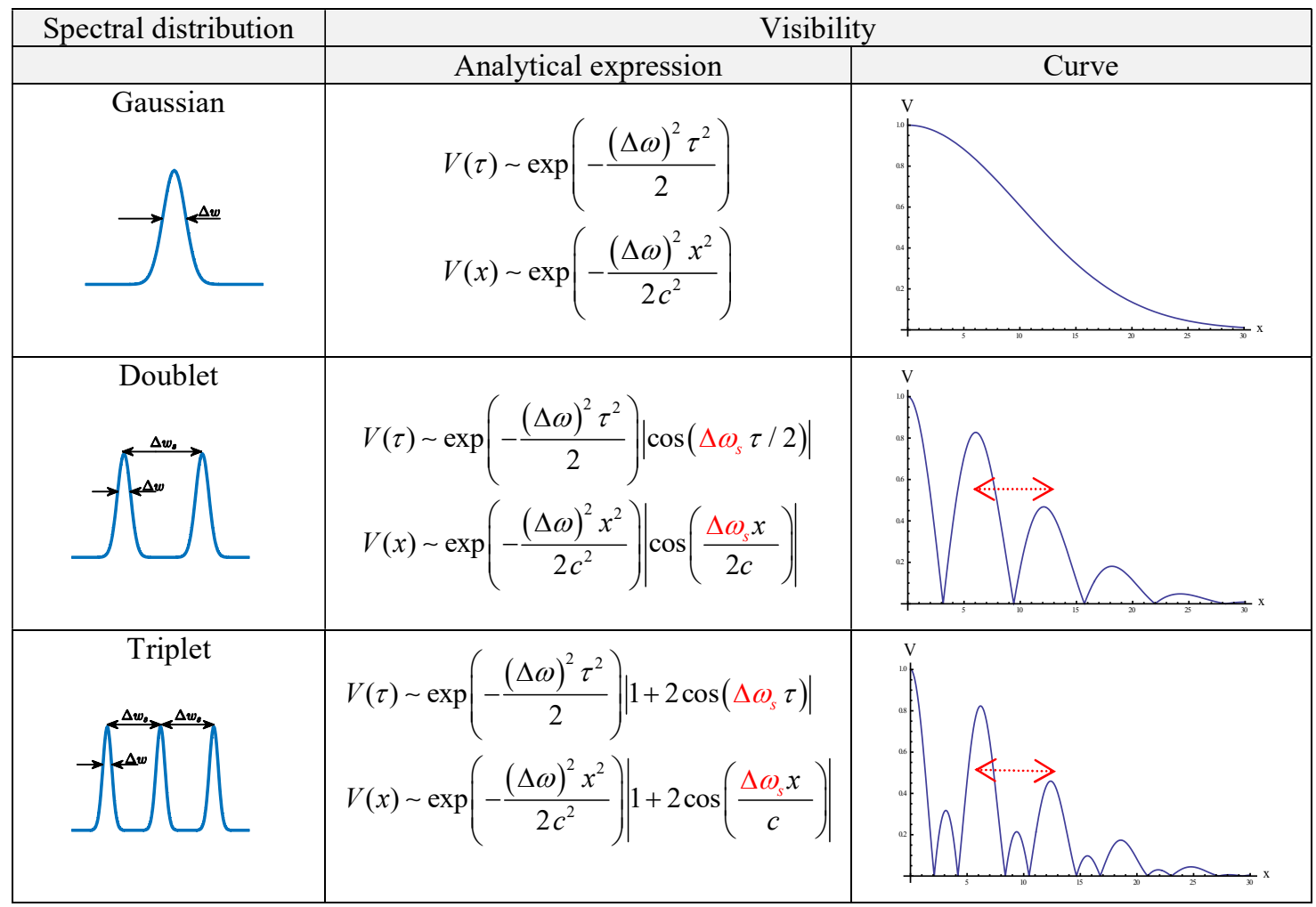


If the spectrum of the beam $I(\omega)$ is very broad in frequency (non-monochromatic light) then $|\gamma(\tau)|$ will be very narrow in time, that is to say, the light beam will be coherent only for small values of $\tau$. Therefore, it will be difficult to see interference fringes. In contrast, if the spectrum $I(\omega)$ is very narrow in frequency (monochromatic light) then $|\gamma(\tau)|$ will be very broad in time and there will be coherence even for large values of $\tau$. Consequently, it will be easy to see interference fringes. For example, if $I(\omega)$ has a Gaussian spectral distribution centered on $\omega_{0}$ and with width $\Delta \omega$, the fringe visibility calculated from Eq. (5) will have the shape of another Gaussian function with a temporal width proportional to $1 / \Delta \omega$ (see the first row in Table I). This temporal width $\Delta \tau$ is often known as coherence time and it represents the maximum time that a wave remains coherent with itself. Thus, the coherence time is inversely proportional to the spectral width of the light source. For practical purposes, the temporal coherence is usually described in terms of the coherence length $\left(l_{\mathrm{c}}\right)$, which is the distance, measured in the direction of wave propagation, that the wave maintains coherence at a fixed instant. The coherence length can be calculated as follows:

$$
l_{c}=\Delta x=c(\Delta \tau)=\frac{c 2 \pi}{\Delta \omega}=\frac{\lambda^{2}}{\Delta \lambda}
$$

Several analytical visibility expressions and visibility curves corresponding to different spectral distributions have been displayed in Table I. In the case of the Michelson interferometer, the variable $\tau=t_{2}-t_{1}$ is replaced by the optical path difference of the interfering waves, which we will call $x$. As can be seen in Table I, as the number of laser modes increases, the visibility curve corresponding to a single-mode laser is modulated by a function depending on the laser-mode separation $\Delta \omega_{\mathrm{s}}$. This magnitude can be estimated from the separation between two maximums of visibility. Therefore, by measuring the distances at which the visibility reaches some minimum value and the distances between two maxima of visibility, we can determine the coherence length and the laser-mode separation, respectively.

\section{EXPERIMENTAL SET-UP}

Once the interferometer shown in Fig. 1 is set and aligned, the interference fringes can be seen on a screen placed on the optical bench. To achieve more accurate results, the beam is expanded and collimated by means of a Galileo telescope. In this stage, it is convenient to set the interferometer at zero optical path difference, $x=0$. The optical path difference can be easily measured on the millimetric optical bench. In our set-up, the screen is replaced by a monochrome electronic camera DMK4IAU02 from Imaging Source [6]. The camera has a CCD detector of $1024 \times 768$ pixels, each of $4.65 \mu \mathrm{m}$ in size, and it is connected to the computer via a USB cable. The camera is controlled by an acquisition program called IC Capture, which is a comprehensive software package capable of capturing uncompressed image sequences and of saving them as video files or as a series of individual bitmaps. Before switching on the camera, some attenuation filters have to be inserted in order to avoid the saturation of the camera. Both the exposure time and the gain in the IC Capture program affect the image of the interference pattern (see Fig. 2), so they have to be adjusted until the appropriate pattern is obtained. It is very important to obtain unsaturated fringes. It may be helpful to have the histogram tool activated in this stage. Measurements should be carried out with small gains and adequate exposure times to avoid saturation. When the interference image obtained is satisfactory, it can be saved. In this exercise, it is enough to save the interference pattern as an image.

The value of the visibility in the acquired images can be obtained in different ways. Any graphical editor capable of measuring brightness can be used. A recommended option is to use the open Java-based image-processing program ImageJ developed at the National Institutes of Health [5].This program, among its many and diverse menu options, allows us to obtain, graphically and numerically, the photometric distribution along any line drawn on the image (see Fig. 3). The line must be drawn as perpendicular as possible to the interference fringes in order to avoid losing visibility, and its thickness can be varied to smooth the calculated photometric distribution.

The calculation of the visibility is done by applying Eq. (3) with the following values: the value of $I_{\max }$ is directly taken from the central maximum, while the value of $I_{\min }$ is the average of the irradiances adjacent to the maximum, i.e. $I_{\min , 1}$ and $I_{\min , 2}$ (see Fig. 3). If the irradiance of the background $\left(I_{\mathrm{back}}\right)$ were too high, the calculation of the visibility would need to be corrected as follows: 


$$
V=\frac{I_{\max }-I_{\min }}{I_{\max }+I_{\min }-I_{b a c k}}
$$
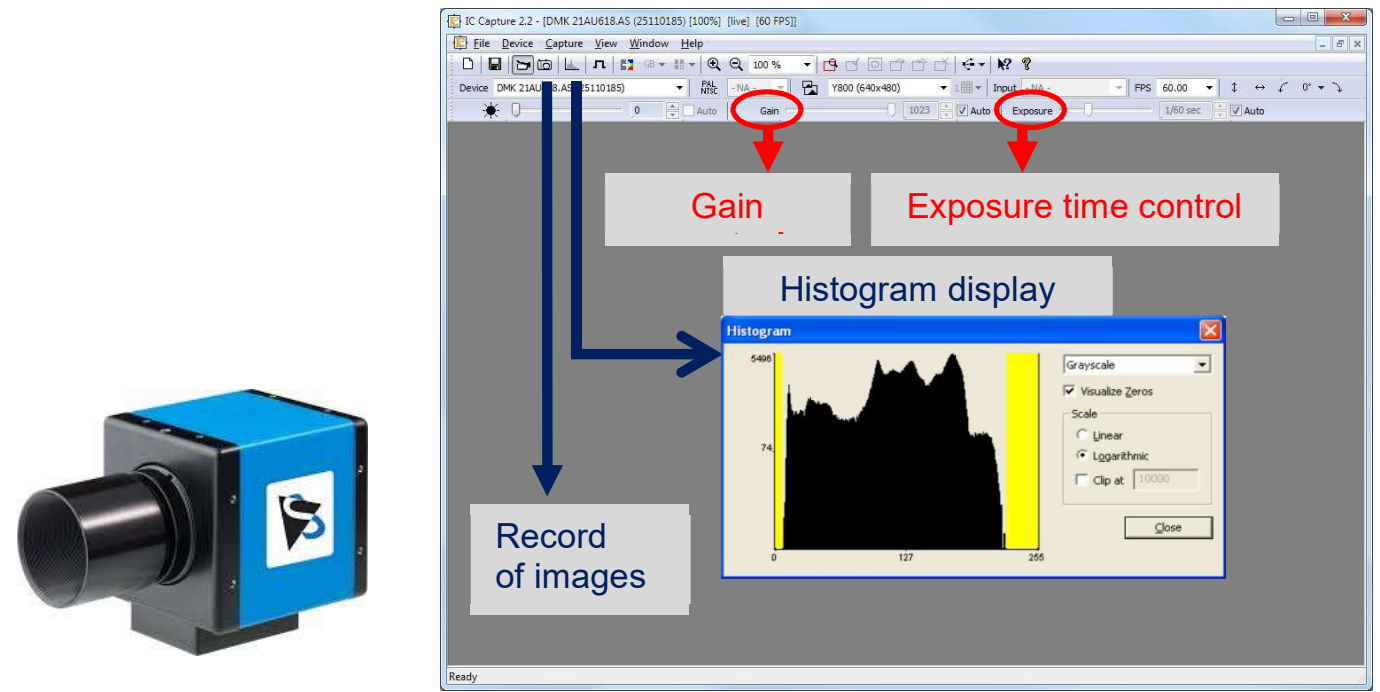

Figure 2. DMK camera and IC Capture program's interface with highlighted menu items.

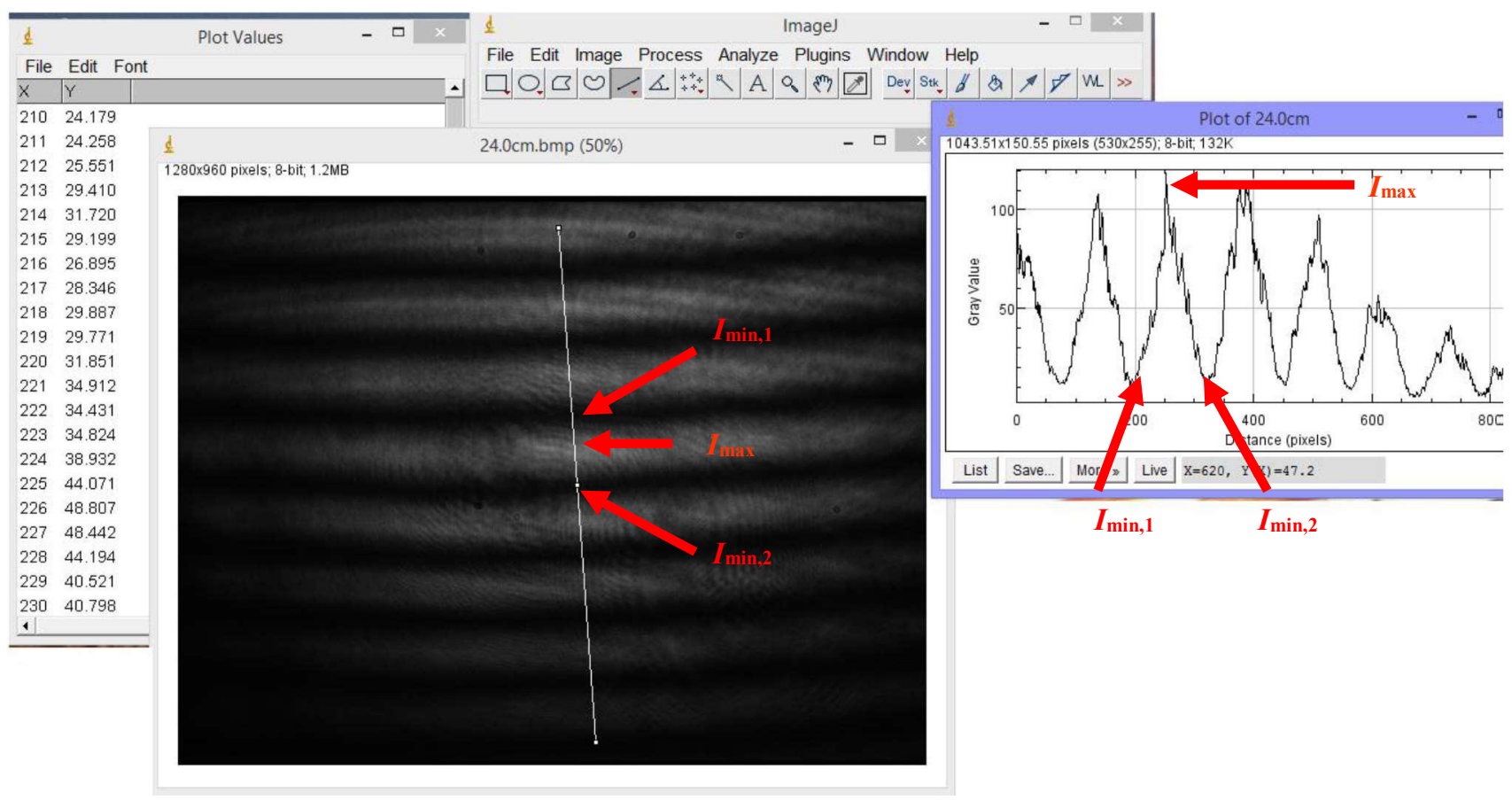

Figure 3. Options of the image-processing program Image $J$ and illustration of the calculation of the visibility from the captured photometric distribution. 


\section{RESULTS}

The results shown here correspond to two different lasers: a He-Ne red laser $(\lambda=632.9 \mathrm{~nm}, 2.5 \mathrm{~mW})$ and a diode-pumped solid state (DPSS) green laser $(\lambda=532 \mathrm{~nm}, 1 \mathrm{~mW})$. From the laser specifications in the manufacturer's data sheets, we know that the He-Ne laser is a two-mode one with a frequency difference of $1500 \mathrm{MHz}$ between both modes, while the DPSS laser is a two- or three- mode laser. Figures 4 and 5 show the visibility curves obtained from both lasers. As an example, we have also displayed the photographs of the interference patterns formed by each laser at two optical path differences corresponding to a maximum and a minimum of visibility.
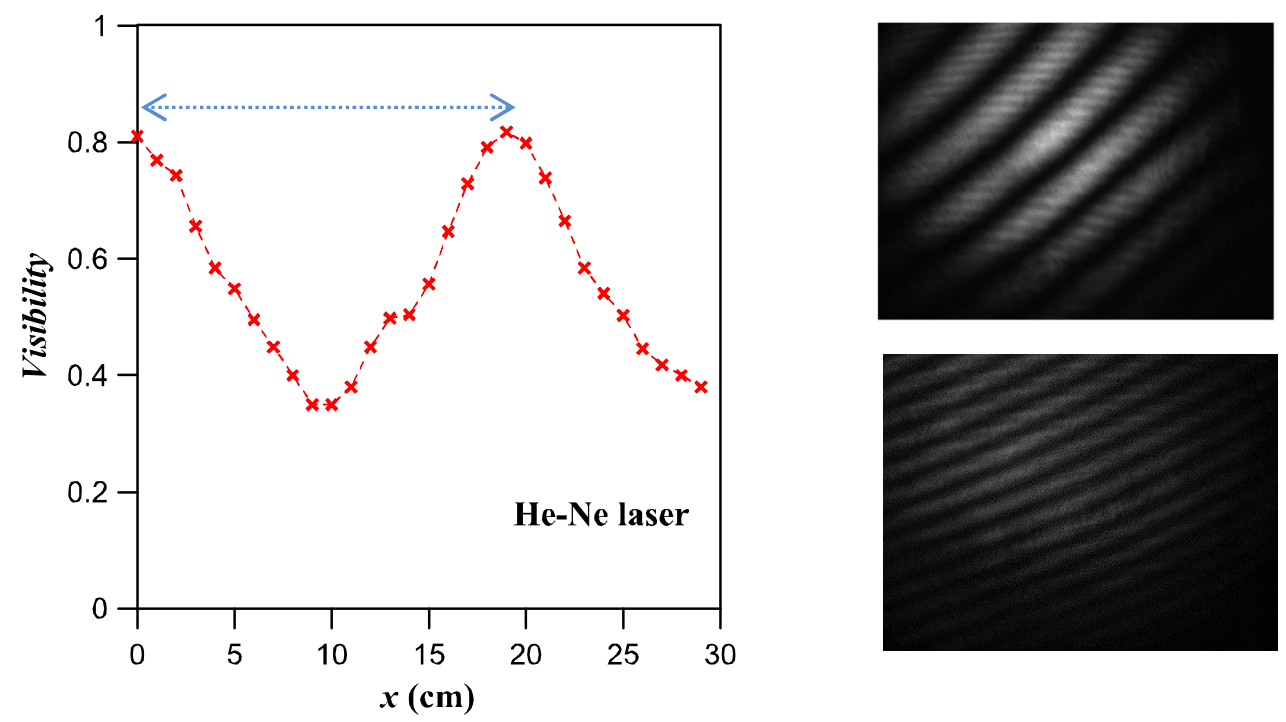

Figure 4. Left: Visibility curve as a function of the optical path difference (error in the data: 5\%). Right: Interference fringe patterns corresponding to two distances $x=0 \mathrm{~cm}$ (maximum $V$ ) and $x=9.5 \mathrm{~cm}$ (minimum $V$ ). The arrow indicates the distance between two maxima of visibility.
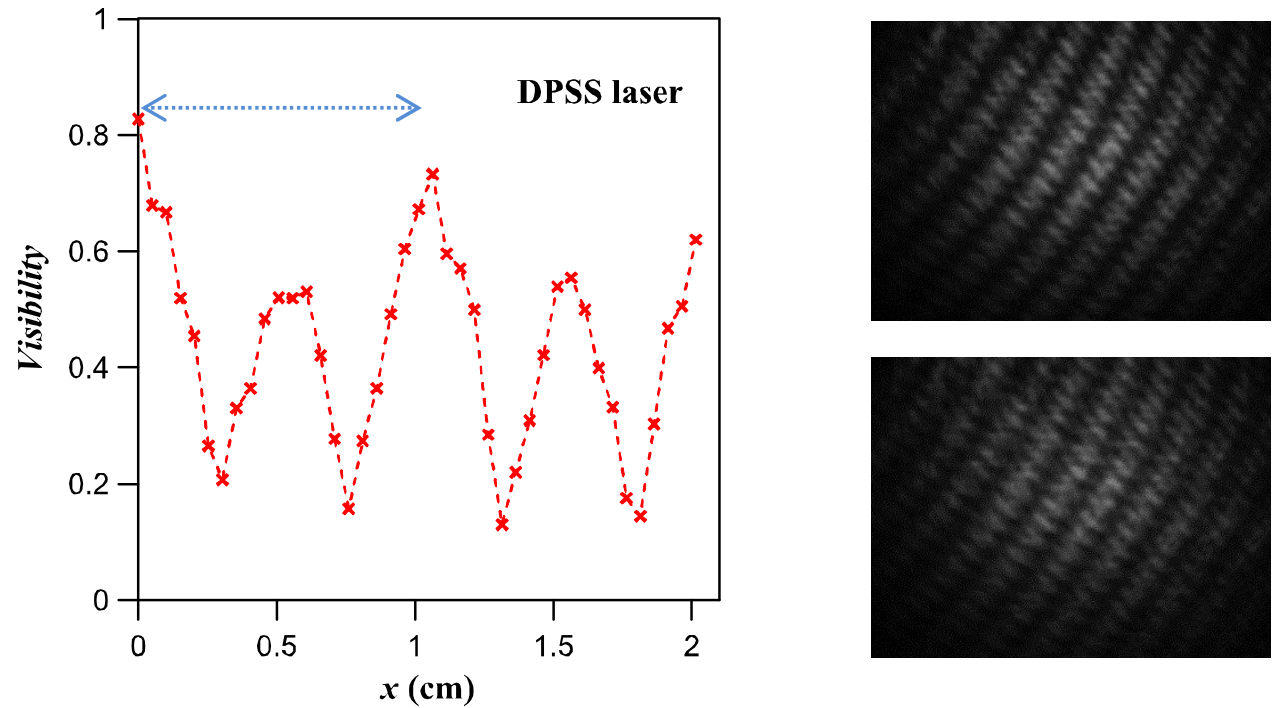

Figure 5. Left: Visibility curve as a function of the optical path difference (error in the data: 5\%). Right: Interference fringe patterns corresponding to two distances $x=0 \mathrm{~cm}$ (maximum $V$ ) and $x=0.3 \mathrm{~cm}$ (minimum $V$ ). The arrow indicates the distance between two maxima of visibility. 
As can be seen in Figs. 4 and 5, the visibility of the He-Ne laser reaches its first minimum value at a distance of approximately $9.5 \mathrm{~cm}$ while the DPSS laser reaches it in a much shorter distance, in about $0.3 \mathrm{~cm}$. These values represent the coherence lengths of the two lasers. Figure 6 illustrates the temporal coherences of the two lasers employed. It can be seen that the He-Ne laser maintains its temporal coherence along a longer distance than the DPSS one. Specifically, the coherence length of the He-Ne laser is one order of magnitude higher, which indicates that He-Ne laser is much narrower spectrally. On the other hand, we can see that the form of the visibility curves shown in Figs. 4 and 5 agree with the specifications of the lasers given by the manufacturer (see rows 2 and 3 of Table I), that is to say, the He- $\mathrm{Ne}$ laser emits with two modes and the DPSS laser with three. From the measured distance between two maxima of visibility, we have obtained that the frequency difference between modes is $1578 \pm 200 \mathrm{MHz}$ for the He-Ne-laser (the nominal value is 1500 $\mathrm{MHz}$ ) and that it is $27273 \pm 5000 \mathrm{MHz}$ for the DPSS laser.

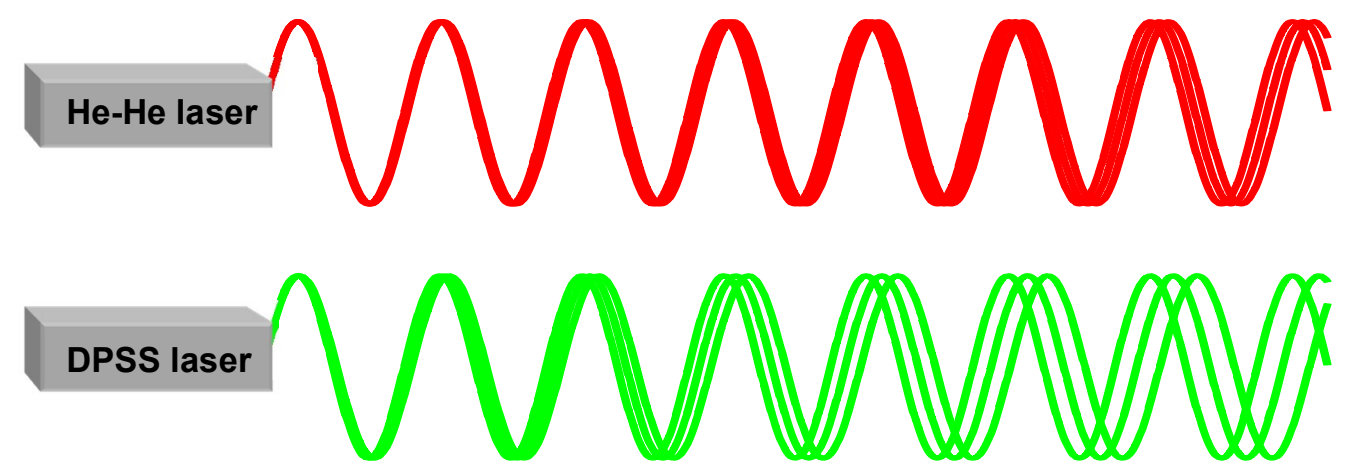

Figure 6. Illustration of the different coherence lengths of the two lasers analyzed.

\section{CONCLUSIONS}

A laboratory exercise has been developed for a better understanding of the temporal coherence of light sources. The experimental set-up consists of a digital camera coupled to a Michelson interferometer. The use of the camera allows our students to quantitatively measure the evolution of the contrast of the interference patterns as a function of the optical path difference of the beams. The analysis of the results has provided the coherence lengths and the frequency difference between laser modes for the two lasers considered. By performing this laboratory exercise, the students will gain insight into some physical principles, such as interference phenomena, concepts of temporal coherence and coherence length, and the relation between the temporal coherence of a light source and its spectral line profile. In addition, they will improve in some experimental skills, such as, handling, alignment and measurement techniques in interferometers, and particularly, in detection with digital cameras and image processing.

\section{ACKNOWLEDGMENTS}

This work has been supported by the following institutions: Ministerio de Economia y Competitividad under projects TEC2015-638263-C03-1-R, Gobierno Vasco/Eusko Jaurlaritza under projects IT933-16 and ELKARTEK (KK-2016/0030 and KK-2016/0059) and by the University of the Basque Country (UPV/EHU) through programs UFI11/16.

\section{REFERENCES}

[1] Born M. and Wolf, E., [Principles of Optics] Pergamon Press, Oxford (1980).

[2] Hecht E. and A. Zajac, A. [Optics] Addison Wesley Publishing Company; 3rd edition (1997).

[3] Pedrotti F.L., Pedrotti L.M. and Pedrotti, L.S., [Introduction to Optics] Pearson, 3rd edition (2007).

[4] Master in Space Science and Technology http://www.ehu.es/aula-espazio/english/master.html

[5] ImageJ https://imagej.nih.gov/ij/

[6] https://www.theimagingsource.com 